\title{
Puesta en valor del Palacio-Castillo de Betxí, a través de la modelización mediante arquitecturas inversas y documentación gráfica avanzada
}

\author{
José Teodoro Garfella Rubioa, María Jesús Mañez Pitarch ${ }^{b}$, Joaquín Ángel Martínez \\ Moya $^{c}$, Francisco Ortega Tomas ${ }^{\mathrm{d}}$ \\ Universitat Jaume I, Castellón, España, ${ }^{a}$ garfella@uji.es, bmanez@uji.es I' ${ }^{c}$ jomoya@uji.es, ${ }^{\mathrm{d}}$ al121041@uji.es.
}

\begin{abstract}
Betxi's Castle Palace is located in the territory of la Plana de Castellon, with a Renaissance-Mannerist character, is a clear example of the evolution and different use of this type of palatial defensive constructions in the Levante Region over time. It has a square floor shape and four towers or defensive bastions on the corners; it is attributed to Joan de Ambuesa Master. It has survived until today, as other Castle-palace as Alaquas and Albalat dels Sorells at Valencia Province, Geldo at Castellón Province or Cocentaina at Alicante Province, among other ones; nevertheless another palaces of that era, now are totally disappeared as Oliva's Palace.

In this case the building has surviving in part over time, adapting to new uses, urban evolution and speculative processes. Luckily currently the property is of municipal ownership and is undergoing restoration and has been documented previously by traditional means. This article aims to give a small brushstroke over part of the work carried out aimed at documenting that property in its current context. For this purpose, it has carried out a rigorous and scientific surveying, without detracting previous graphic documentation works, using advanced documentation techniques, through active and passive 3D last generation imaging systems, as well as topographic support and global positioning systems. On the other hand, in order to concretize the enhancement, it has been modeled and built a series of digital reverse architectures and other ones tangible to represent the whole Castle-palace in their primitive appearance or some details of the building itself, based both on own evidences that still remain in the building and its surroundings, as the ones obtained through bibliography and archival and historical data, published or found related to this building.
\end{abstract}

Keywords: Betxi's Castle-palace, reverse architecture, advanced documentation, Juan de Ambuesa.

\section{Introducción}

Tras la reconquista cristiana del Reino de Valencia por parte de Jaume I, de Aragón, los musulmanes se vieron obligados a abandonar el territorio ocupado dejando sus posesiones, o a convertirse al cristianismo. Tras esta Reconquista, el rey donó las villas, los castillos o lugares conseguidos a los caballeros y órdenes religiosas militares que le ayudaron durante la Reconquista. Así, los propietarios y dueños de los diferentes sitios fueron caballeros, órdenes religiosas militares o el propio rey. En este territorio existían múltiples castillos de origen moriscos e incluso romano que había sido construida por los musulmanes para defenderse de los ataques cristianos. Algunos de estos castillos con el reparto fueron preservados, reconstruidos y adaptados. Mientras que otros fueron construidos de nuevo, para dar cobijo a 
los señores. Este tipo de construcciones se materializaban normalmente en la cima de las montañas, con el fin de defender los vastos territorios que formaron, tratándose de construcciones simples, generalmente con una torre de vigía y una pared exterior, construidos con materiales de la tierra y sin decoración. En el siglo XIII, con la toma de posesión por parte de los cristianos algunos de estos viejos castillos fueron mejorados, con la configuración y lenguaje nuevo con muros mas altos y gruesos, la construcción de barbacanas y fosos, convirtiéndose en auténticas fortalezas desarrolladas sobre las antiguas fortalezas árabes y sobre las que se adosarían y posteriormente se desplegarían las poblaciones. Después de un período de quietud, rey Pedro el Ceremonioso ordenó la renovación de las fortificaciones contra una posible invasión de los moriscos de Granada en el siglo XIV. Desde el siglo XV los edificios militares se convirtieron en castillos feudales, pero poco a poco los castillos perdieron su sentido defensivo y se convirtió en castillo-palacios. El siglo XV fue la época de esplendor de estas mansiones nobles. Sobre la sociedad tanto como el binomio señor Castillo, que hasta los palacios construidos en la llanura, conservó el nombre de "Castillo", aunque en ocasiones fueron palacios para la residencia del señor sin ninguna pretensión defensiva. Sin embargo, las continuas guerras y levantamientos de los moros (árabes que no ha terminado de convertir al cristianismo), algunos de estos hechos Castillo-Palacios tuvieron que fortificar, hasta que en el año 1609 Felipe II ordenó la expulsión.

\section{Características del Castillo Palacio}

Se trataba de un edificio de planta cuadrada flanqueada por torreones en las esquinas y rodeado por un foso. El edificio conserva dos plantas La fachada es de treinta y dos metros de largo y su altura muy variable. En el interior queda poco más de medio patio con galerías claustrales por los cuatro lados. La base de la actual edificación es gótica, con una importante fase en el siglo XVI y con el añadido de finales del siglo XIX y principios del XX. Se sabe de su existencia ya en el siglo XIV como construcción militar que protegía la acequia y en la que tenían defensa y refugio las tropas romanas que guarnecían la Plana. Al mismo tiempo servía de almacén para cereales, vino y aceite que se exportaban a Roma y Oriente por el puerto de Burriana. Hacia el siglo XVI se adaptaría el palacio al estilo renacentista sobre la edificación gótica ya existente. La huella renacentista se puede apreciar en la puerta de acceso al palacio, en la puerta del patio de medio punto y en las columnas y arcos del patio, pero conservando elementos góticos tales como el arco de entrada al patio o las ventanas ajimezadas desaparecidas sobre la década de 1960. Se atribuye a Juan de Ambuesa, la autoría de la intervención. El edificio se conservó, con altos y bajos, en su estado hasta la extinción de los mayorazgos por los años veinte del siglo XIX. Fue entonces cuando dejó de interesar a sus señores, quedando en completo abandono hasta ser convertido en cuartel provisional de las tropas portuguesas que defendieron el trono de Isabel II durante la primera Guerra Carlista. Años después, fue vendido a varios vecinos que se asociaron para la compra y explotación del mismo y se derribaron cubiertas, muros, suelos y se vendieron sus materiales. Posteriormente fue adquirido, a finales del siglo XIX, se dividió la propiedad del palacio por uno de los lados del patio y se cegó la arquería renacentista que hace las veces de medianera. Se le añadió un edificio a su izquierda a la moda de la época, de planta baja y dos pisos, con pinturas al fresco, escalera modernista y vidrieras art déco.

La mejor época del palacio de Betxí fue de 1510 a 1571 , durante estos años se realizarían las obras de la portada principal y del patio, las más importantes del monumento y, por su traza, se estiman más antiguas que los torreones y las fortificaciones. La portada, de traza muy italiana con arco de medio punto y pilastras toscanas, se adorna con almohadillados y finas molduras. Sin duda alguna, la obra cumbre es el patio. Su acertada disposición, trazado, adecuada modulación y elementos decorativos, forma un conjunto notable que, como la portada, no quedaron terminados y poco le ha acompañado la suerte de su aprovechamiento y conservación. 
Actualmente se está recuperando e interviniendo sobre el referido inmueble. $\mathrm{Al}$ respecto del edifico se han realizado previamente por otros equipos técnicos trabajos de aventamiento $\mathrm{y}$ documentación previa del edifico incluso con tecnologías de última generación

\section{Objetivos}

La presente, entre otras, pretende a partir de la inquietud que han generado los nuevos métodos y sistemas de documentación avanzada del patrimonio dar una pequeña pincelada e ilustrar la presente comunicación. Por otro lado se pretende, avanzar o profundizar en el alcance y utilidad de estas herramientas que pueden optimizar los resultados de los trabajos gráficos poniéndolos en valor a través de arquitecturas inversas, modelos $3 \mathrm{D}$, video etc..., sin que estos pierdan rigurosidad ni desvirtúen la realidad en detrimento de la calidad del trabajo a desempeñar.

Para ello compararemos algunos de los sistemas consabidos basados en sensores tridimensionales activos como son el láser escáner 3D terrestre de mayor coste y menos accesibles a un público en general con el empleo de las herramientas de software gratuitas o de low cost, basados en sensores tridimensionales pasivos de imagen o los que nosotros conocemos como Fotogrametría Digital Automatizada o por las siglas en ingles SFM (Structure for Motion), buscando la sostenibilidad y el enriquecimiento de la expresión gráfica al alcance de cualquier persona u operador.

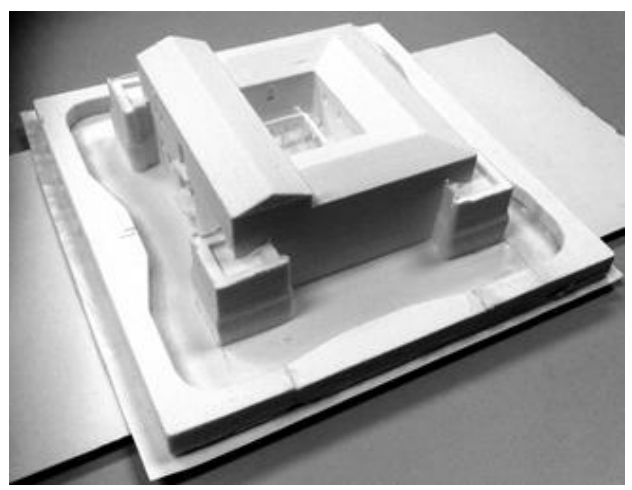

Fig. 1- Impresión 3D Castillo Palacio.

\section{Metodología}

Para poder materializar la documentación gráfica y el levantamiento arquitectónico deseado se ha procedido a la realización de una serie de tareas, que van desde lo mas artesanal como son la elaboración de croquis hechos a mano y medición directa con el empleos de medios y recursos tradicionales como son la cinta métrica el fluxómetro y el peine perfilador, al empleo de tecnologías de ultimas generación basado fundamentalmente en la captación mediante sistemas activos de imagen 3D de tipo pasivo asociados a la toma de fotografías y posterior posproceso y de sistemas activos de imagen 3D mediante el empleo del escáner laser terrestre, concretamente un escáner terrestre 3D basado en pulsos, de láser invisible clase uno de largo alcance y precisión de $4 \mathrm{~mm}$ en un rango de escaneo hasta $150 \mathrm{~m}$ y angular de 6" (2,0 mgon), asociado con una cámara digital de 2.0 Mega píxeles integrada, alineada coaxialmente.

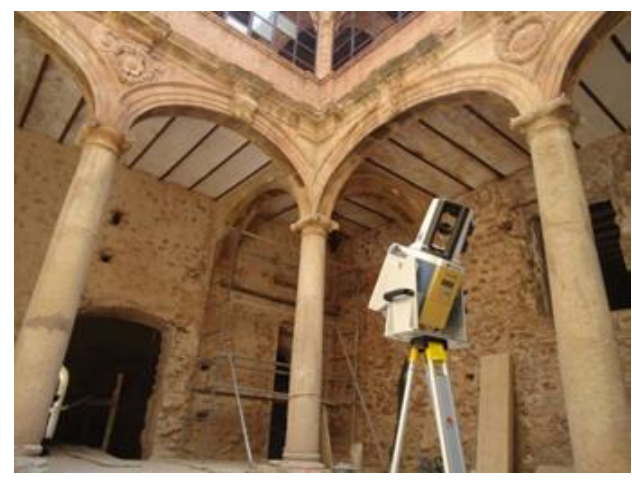

Fig. 2- Escaneo zona del patio.

También se ha completado la toma de datos con dos cámaras fotográficas digitales réflex de 10,2 Mega píxeles, y de 24 Mpx respectivamente. Con un objetivo convencional comprendido entre los $18-135 \mathrm{~mm}$. y con apertura focal de f/3.5-5.6. y un gran angular con un objetivo 8 $16 \mathrm{~mm}$. y apertura focal de f/ 4,5 - 5,6. Previamente a la realización de las fotos de campo, se ha procedido a la calibración de las cámaras para la detección la corrección y distorsión de las cámaras, mediante software

Con el escáner 3D se han tomado varias nubes de puntos (escaneados) con una densidad 
aproximada de $4 \times 4 \mathrm{~mm}$. a $15 \mathrm{~m}$., para lo cual se han realizado varios estacionamientos y a su vez uno o varios escaneados de cada uno de los estacionamientos incluido un barrido de dianas en cantidad superior a tres por estación, para su posterior enlace $u$ orientación. Con el emplazamiento de las estaciones y con los distintos barridos, se ha pretendido sortear las dificultades o sombras producidas por los propios elementos o mobiliario del edificio.

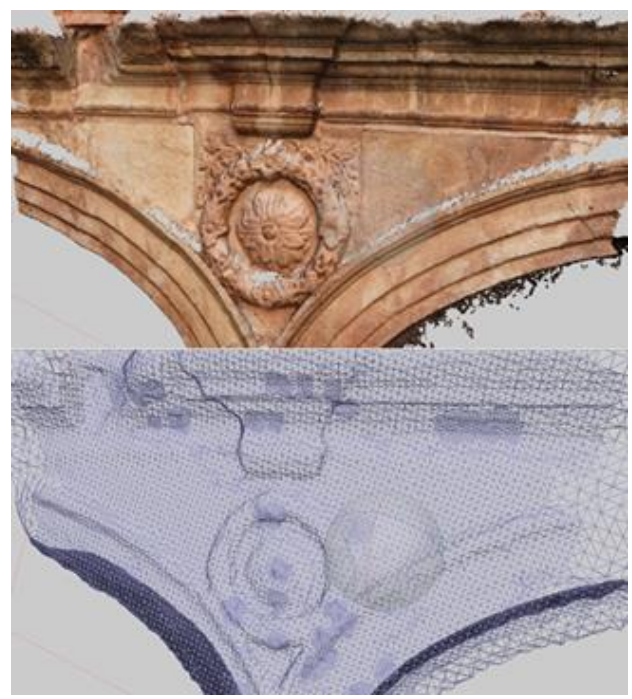

Fig. 3- Modelización textura y mallado fotogramétrico.

Desde cada estación se ha tomado unas nubes de puntos en general, y otras de detalle con distintas densidades hasta alcanzar como máximo los 30.000 puntos por segundo prestación máxima del equipo,

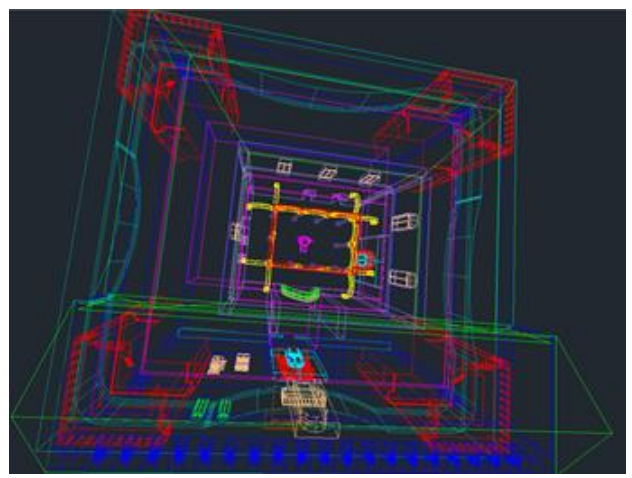

Fig. 4- Modelización y vectorización en CAD.
Las mediciones o toma de datos estándar se ha realizado con el compensador angular de dos ejes $(x, y)$ de forma automática, mientras que el resto de posiciones angulares se han realizado deshabilitando el dispositivo automático de compensación angular.

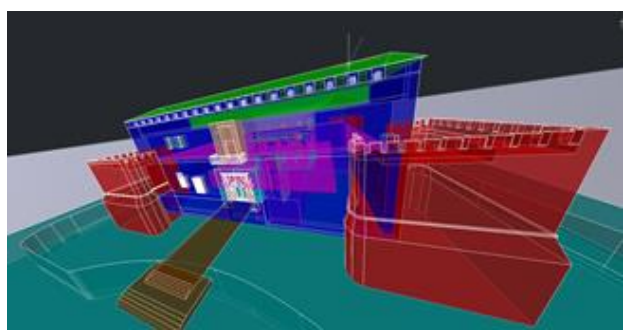

Fig. 5- Perspectiva y vectorización en CAD

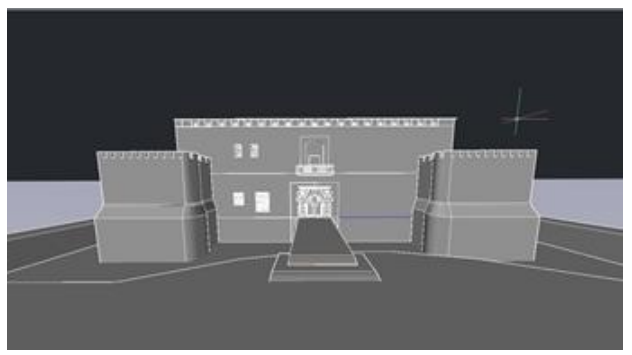

Fig. 6- Perspectiva y modelado en CAD

De este modo de cada uno de los puntos se obtienen las coordenadas ( $\mathrm{x}$ y $\mathrm{z}$ ), índice de intensidad de color (RGB). Esto se refiere a la intensidad de retorno del rayo láser, según muestran los colores rojo, verde y azul. Las superficies que son más oscuras y tienen menor intensidad de retorno aparecen de color azul; las superficies con intensidad de retorno media se presentan de color verde; y las superficies con intensidad de retorno alta se muestran de color rojo, así como la intensidad del rayo de reflexión. Con la cámara fotográfica asociada al escáner 3D se han obtenido fotografías del modelo que quedan automáticamente referenciadas respecto a la nube de puntos, dado que la cámara forma parte del propio escáner o estación de imagen, de tal manera que el software a emplear en el postproceso reconoce para cada una de las fotografías la dirección del eje óptico de la cámara y el ángulo de visión, dado que se emplea un objetivo de focal fija. Todo esto permite asociar el color del pixelado 
fotográfico a los puntos obtenidos con el escáner y también mapear sobre la triangulación de la nube de puntos las fotografías tomadas, obteniendo así un modelo virtual del objeto representado.

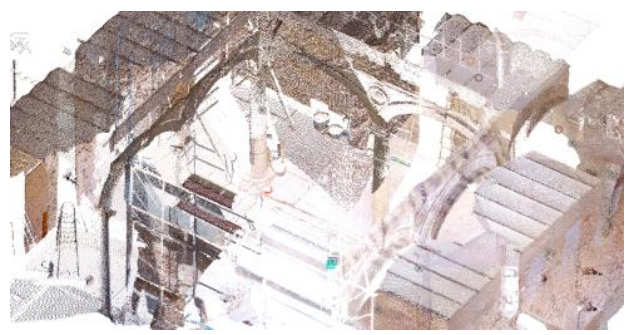

Fig. 7- Perspectiva nube de puntos escáner.

En cuanto al postproceso o trabajo de gabinete se ha empleado como herramienta de trabajo, básicamente los software de los equipos empelados los cual gestionan las nubes de puntos, permite visualizan, navegar, medir y trabajar con los modelos de datos tridimensionales obtenidos a través del escáner. Procediendo al registro de las distintas nubes de puntos mediante las dianas retroreflectantes y los puntos fijos establecidos, lo que permite unir las nubes de puntos parciales, tomadas desde las distintas posiciones, en una nube de puntos general, alcanzando una definición completa del modelo evitando en lo posible las zonas de sombras, enlazándolas entre si y procediendo al modelado en su caso de formas tridimensionales.

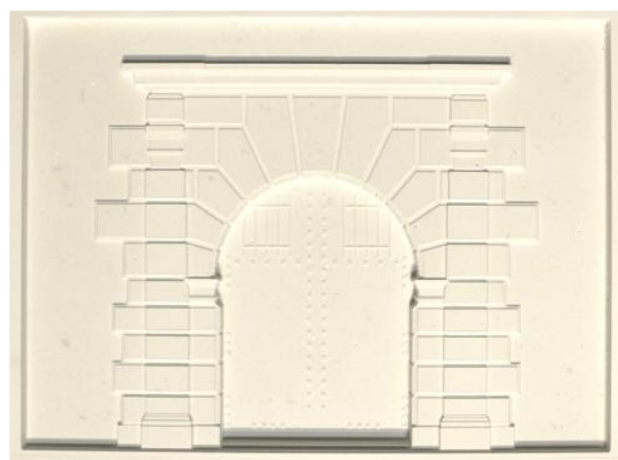

Fig. 8- Fresado de puerta con control numérico.

Por otro lado los programas integran toda la información obtenida de las estaciones de imagen, los apoyos topográficos obtenidos con la Estación Total y las fotografías realizadas con las cámaras fotográficas externas. Procediendo a su fusión creando superficies texturizadas que se procesan como una Malla Irregular Triangulada (TIM), completando las lagunas o sombras mediante la fotografía y se vuelven a procesar para obtener un modelos estereoscópicos o tridimensional que nos permite la toma de medidas, la realización de ortofotos, crear isolíneas, perfiles transversales, etc.

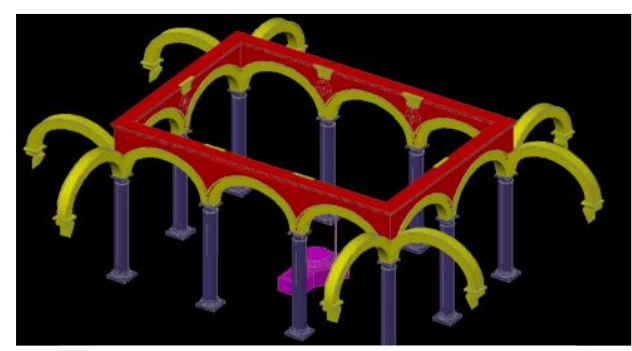

Fig. 8- Perspectiva con dibujo 3D patio central

Por último los datos medidos y las ortoimágenes se pueden extraer en formatos compatibles de trabajo como son los formatos DXF, JPEG, LAS y OBJ. Con el formato antes citado se puede comenzar la vectorización mediante software grafico específico, a la vez que se puede configurar las mallas tridimensionales o TIM para posprocesar con programas de modelado el objeto y obtener un modelo digital tridimensional texturizado o no, en formato VRML.

Tras ello se puede imprimir en modelo digital con una impresora física 3D, o proceder al fresado de un elemento, mediante el empleo de máquinas de control numérico.

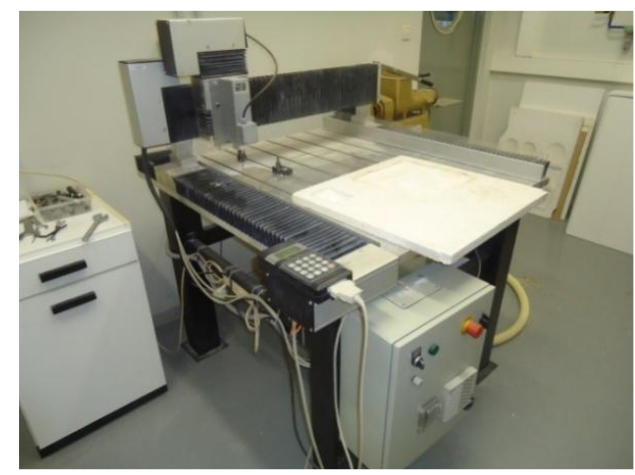

Fig. 9- Fresadora de control numérico 


\section{Agradecimientos}

Agradecemos a la Universitat Jaume I y al Departamento de Ingeniería de Sistemas Industriales y Diseño, área de expresión gráfica arquitectónica por su colaboración y la dotación necesaria para poder realizar este trabajos y al Ayuntamiento de Betxi por su atención y las facilidades prestadas.

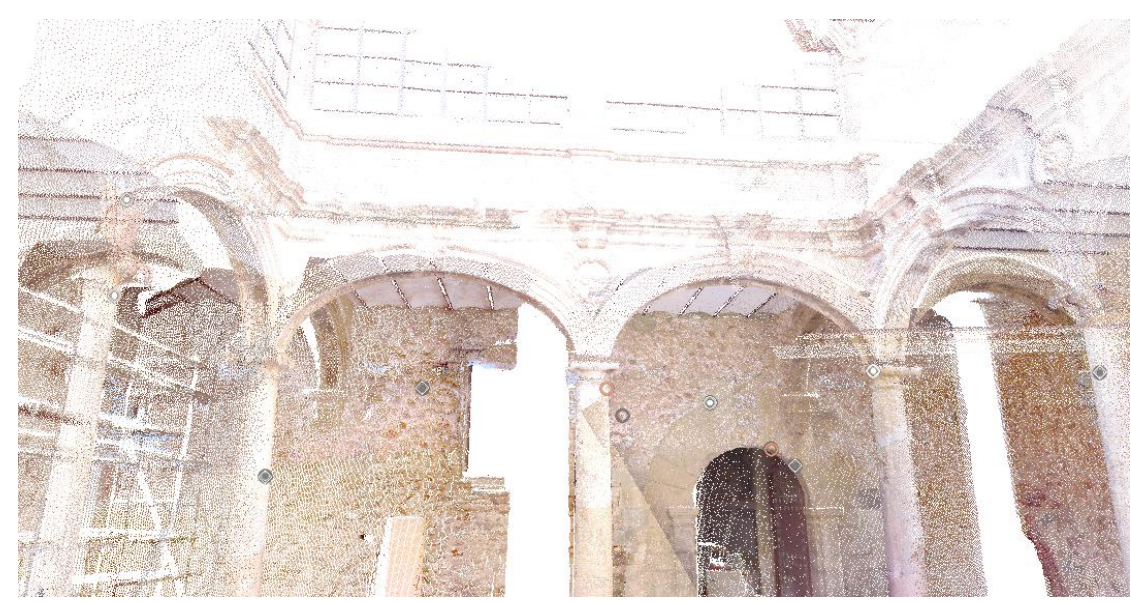

Fig. 10- Nube de puntos general del patio central a través del escáner laser 3D.

\section{Referencias}

Albiac i Mesado, Vicent. (2003-2005). En Pascual Meneu i Meneu. Estudis Calellonencs, Diputació de Castelló, 10. pp. 735-740.

Berchez, Joaquín y Jarque, Francesc. (1994). Arquitectura renacentista Valenciana, 1500-1570, Valencia, 104 y ss.

Gómez Ferrer Lozano, Mercedes. (1998). Arquitectura en la Valencia del siglo XVI. El Hospital General y sus artífices. Valencia: Albatros.

Llop Vidal, Enric, Grande Grande, Francisco y Francisco Juan Vidal. (2003). Memoria del Plan especial del Palau Castell.

Mesado i Ximeno, Xavier. (2003-2005). El somni de Pascual Meneu: El Palau de Betxí. Estudis Calellonencs, Diputació de Castelló, 10. pp. 741 - 748.

Mesado Oliver, Norberto. (2003-2005). D. Pascual Meneu i Meneu. Un "Betxinenc" ilustre. Estudis Calellonencs, Diputació de Castelló, 10. pp. 749 - 880.

Palaia, Liliana y Tormo, Santiago. (2008). Informe final. Estúdio construtivo. Palacio de los condes de Ariza, Betxí, Castellón.

Palaia, Liliana y Tormo, Santiago. (2009), El palacio de Betxí. Historia de su construcción a través de la lectura del edificio, Actas del sexto Congreso Nacional de Historia de la construcción.Valencia. Eds S. Huerta, R. Marín, R.Soler, A. Zaragoza. Madrid: Instituto Juan de Herrara. pp. 1019 - 1028.

Serlio, Sebastiano. [1552] (1986), De todas las obras de arquitectura y perspectiva de Sebastian Serlio de Bolonia. Edición facsimilar. Oviedo: COAAT de Asturias.

Tamborero Capilla, Lourdes y Martínez Porral Rafael. (2008), Memoria de las Intervenciones Arquelógicas del Palau-Castell de Betxí, (2 ${ }^{\mathrm{a}}$ Fase).

Traver, Vicente. (1961), "El palacio-castillo de Bechí”. Boletín de la Sociedad Castellonense de Cultura, 37, Cuaderno IV, pp. 253 - 67.

Zaragoza Catalán, Arturo. 1991. La casa señorial valenciana. «Palau de l'Almirall, Valencia», pp. 81 94.

Zaragoza Catalán, Arturo. 2000. Arquitectura Gótica Valenciana. Valencia. 